\title{
EDITORIAL
}

\section{Optimisation of cardiac resynchronisation therapy: addressing the problem of "non-responders"}

\author{
D J Fox, A P Fitzpatrick, N C Davidson
}

Heart 2005;91:1000-1002. doi: 10.1136/hrt.2004.043026

Cardiac resynchronisation therapy has become firmly established as a treatment for patients with symptomatic heart failure. Several randomised controlled trials and numerous observational studies have demonstrated improvements in exercise capacity and quality of life. Despite these advances it is clear that approximately $25 \%$ of patients who meet current criteria for implantation of such a device do not show objective evidence of clinical benefit. Implantation of a CRT device is expensive, time consuming and involves some risk so it is important to accurately identify patients who are likely to respond and to optimise pacing lead placement and device programming to maximise the benefit in these selected patients.
See end of article for authors' affiliations

Correspondence to: Dr David J Fox, Manchester Heart Centre, Manchester Royal Infirmary, Oxford Road Manchester M13 9WL, UK; david.j.fox@talk21. com
D isruption of the usual sequence of ventricular activation is now recognised as a major factor in the development of symptomatic heart failure in patients with ischaemic and non-ischaemic cardiomyopathy. Delayed and dyssynchronous left ventricular (LV) contraction reduces myocardial efficiency, causes abnormal diastolic interactions between the ventricles, $^{1}$ and increases mitral regurgitation. ${ }^{2}$ Biventricular pacing, also known as cardiac resynchronisation therapy (CRT), is designed to reduce symptoms of breathlessness and fatigue in these patients by restoration of a more physiological sequence of cardiac activation.

Early clinical trials of CRT were carried out in the USA and Europe from 1995 to 1998 using a thoracoscopic procedure for lead placement on the epicardial surface of the lateral wall of the left ventricle. ${ }^{3}$ In 1998 Daubert and colleagues ${ }^{5}$ reported a technique of transvenous lead implantation with manipulation of a lead into a cardiac vein via the coronary sinus to enable pacing of the epicardial surface of the left ventricle. However, despite further developments, this technique remains challenging and time consuming with average procedure times in large clinical trials of between 2-4 hours. Although implantation success rates for LV leads have risen with greater experience, in most series they do not exceed $90 \% .{ }^{6}$ Moreover, this is a relatively expensive treatment which carries significant risks when performed in patients with heart failure and incipient pulmonary oedema. ${ }^{7}$
THE PROBLEM OF "NON-RESPONDERS"

Important effects on the functional status of the patient are difficult to quantify objectively and very significant placebo effects can be seen with this type of intervention. The functional assessments therefore need to be performed in conjunction with less subjective measurements of ventricular performance and exercise capacity, including serial echocardiography and maximal oxygen uptake $\left(\mathrm{VO}_{2} \max \right)$ pre- and post-CRT. Most patients who receive CRT will have an improvement in their symptomatic status. Trial data have demonstrated an overall mean increase in $\mathrm{VO}_{2}$ max of approximately $1-2 \mathrm{ml} / \mathrm{kg} /$ min, in exercise duration of 30-60 seconds, and in six minute walk test distances of $20-40 \mathrm{~m}^{6}{ }^{6}$ Echocardiographic data demonstrate reductions in LV dimensions and reduced severity of mitral regurgitation. ${ }^{8}$ However, the dramatic treatment effect seen in many patients in the clinical trials has consistently been diluted by the $20-30 \%$ of patients who have no objective clinical benefit following implantation of a CRT device. ${ }^{9}$ 10

At this stage in the development of CRT it is important to identify these "non-responders" and to consider the possible underlying reasons. The patient could then be spared an ineffective intervention or the therapy could be optimised to ensure maximal benefit, thus using limited healthcare resources more efficiently. In practice this process will involve:

- improvement in the assessment of patients before to CRT

- optimisation of pacing lead positions

- optimisation of device programming.

\section{PATIENT SELECTION}

Entry criteria for clinical trials of CRT

The accepted criteria of patient selection for CRT are:

- New York Heart Association (NYHA) class III/ IV symptomatic heart failure

- LV ejection fraction $<35 \%$

- LV end diastolic diameter $>55 \mathrm{~mm}$

- QRS duration $>120 \mathrm{~ms}^{*}$

- sinus rhythm

- stable optimal medical treatment.

Abbreviations: AV, atrioventricular; $C R T$, cardiac resynchronisation therapy; $L B B B$, left bundle branch block; LV, left ventricular; NYHA, New York Heart Association; RBBB, right bundle branch block; TDI, tissue Doppler imaging; $\mathrm{VO}_{2}$ max, maximal oxygen uptake 


\section{Viability of myocardium}

When heart failure is related to ischaemic heart disease, viability of LV myocardium is likely to be a crucial determinant of the response to CRT and patients with extensive LV scarring are unlikely to benefit. This is supported by a study demonstrating that patients with severe resting myocardial perfusion defects had no objective evidence of augmented ventricular function following CRT, in contrast with the improvements seen in patients without resting defects and despite an apparent symptomatic improvement in both groups. ${ }^{15}$ Present practice does not generally include an assessment of LV viability before CRT and further studies are required to determine the role of such assessments in patient selection.

\section{Electrical versus mechanical assessment of dyssynchrony}

An important limitation of initial CRT trials is the use of QRS prolongation (in particular left bundle branch block (LBBB)) on the standard ECG as the sole marker of ventricular dyssynchrony. The trials have included a relatively small number of patients with right bundle branch block (RBBB). In both the MIRACLE and CONTAK $\mathrm{CD}^{16}$ trials small groups of patients with RBBB appeared to respond as well as those with LBBB. Garrigue and colleagues ${ }^{17}$ also demonstrated that patients with RBBB respond to CRT, but only when associated with intraventricular dyssynchrony. The presence of RBBB can mask significant ventricular conduction delay in the left ventricle and echocardiography is required to assess ventricular dyssynchrony pre-implantation. The surface ECG provides a relatively crude assessment of myocardial activation and may not show localised delays which have important mechanical consequences. Several studies have shown that QRS duration is a relatively poor predictor of the symptomatic response to CRT in an individual patient, ${ }^{18} 19$ and there is increasing evidence that patients with ventricular dysfunction and "normal" QRS duration can benefit. ${ }^{20}$ More detailed analysis of ventricular activation with vector cardiography, signal averaged ECG, or non-contact endocardial mapping ${ }^{21}$ may have a role in patient selection.

\section{ASSESSMENT OF MECHANICAL DYSSYNCHRONY Echocardiography}

There are two main echocardiographic predictors of benefit to CRT: firstly, the difference between the aortic pre-ejection period and the pulmonary pre-ejection period estimated by standard pulsed wave Doppler; and secondly, septal to lateral wall contraction delay, best assessed by tissue Doppler imaging.

The larger the difference between the aortic pre-ejection period and the pulmonary pre-ejection period, the greater the chance of response to CRT, which is independent of QRS duration. A delay of greater than $40 \mathrm{~ms}$ is considered compatible with significant dyssynchrony, the larger the delay the greater the chance of response to CRT. ${ }^{22}$

The most widely used technique for identification of mechanical dyssynchrony uses tissue Doppler imaging (TDI), which involves measuring the time to peak myocardial sustained systolic $\left(\mathrm{T}_{\mathrm{S}}\right)$ and diastolic velocity in different segments of myocardium. Typical appearances in patients with heart failure are of a notable regional variation, usually with the earliest activation in the basal anterior septum and the latest in the basal lateral segments. Yu and colleagues ${ }^{23}$ demonstrated mechanical dyssynchrony using TDI in 75\% of those with a QRS > $120 \mathrm{~ms}$, but also in $40 \%$ of heart failure

* Many of the large multicentre trials (COMPANION, PATH CHF, MIRACLE, MUSTIC) adopted a policy of inclusion which ranged from $>120 \mathrm{~ms}$ to $>150 \mathrm{~ms}^{11-14}$ patients with normal QRS duration, demonstrating the weakness of ECG criteria for the accurate detection of dyssynchrony. Three dimensional echocardiography has also been studied and is likely to have a role in these assessments.

\section{Other methods}

Other potential techniques to assess mechanical dyssynchrony include magnetic resonance imaging ${ }^{24}$ and radionuclide studies, but clinical trials are needed to evaluate these prospectively. There may also be a role in patient selection for neurohormonal markers, such as brain natriuretic peptide, which is released predominantly from viable LV myocardium in response to increased LV wall tension. ${ }^{25}$

\section{LEAD POSITIONING AND OPTIMISATION OF PACING SITE}

Butter and colleagues ${ }^{26}$ showed that the LV pacing site was a crucial factor in determining the effects of CRT, with lateral (free wall) sites consistently producing better short term haemodynamic responses than anterior sites. This study showed that in $30 \%$ of patients, stimulation of the anterior left ventricle via tributaries of the great cardiac vein resulted in worsening of haemodynamic function. This can be explained by the fact that LV free wall sites are consistently the latest to be activated and stimulation at these sites reliably restores homogenous LV activation. Conversely anterior wall sites are usually activated early and pacing to advance their activation further will enhance regional dyssynchrony. ${ }^{27} 28$

Pacing of the LV free wall usually means implanting the lead in either the posterolateral, lateral, or anterolateral veins, in the territory supplied by the circumflex or diagonal coronary arteries. With the transvenous approach the operator is constrained by the coronary venous anatomy, which shows considerable inter-individual variability, and the issue is complicated by the inconsistent nomenclature which is used for the veins. It is often difficult or impossible to deliver a lead to the most suitable site of the ventricle. The left phrenic nerve runs down the lateral LV wall and diaphragmatic stimulation can further limit pacing site options. With current lead delivery systems an LV free wall site cannot be achieved in $5-10 \%$ of cases. In the MUSTIC study $^{14}$ a lateral position was achieved in only $80 \%$ of cases, and this may be one reason for the proportion of nonresponders. Improved technology will facilitate lead delivery to a suitable site but in some cases this may be impossible and the above data suggest that it is better to abandon the procedure rather than deploy the lead in a site which may worsen cardiac function. There is a continuing role for surgical epicardial lead implantation when a lateral lead position cannot be achieved by the transvenous route.

\section{DEVICE PROGRAMMING: ATRIOVENTRICULAR (AV) AND VENTRICULAR-VENTRICULAR (VV) DELAYS}

For CRT to work as intended there should be a paced rhythm at all times so the atrioventricular (AV) delay on the pacemaker must be set below the intrinsic AV conduction time. Often the AV delay is programmed at a nominal low value of around $100 \mathrm{~ms}$ to ensure ventricular pacing, but this may excessively reduce the atrial contribution to LV filling and echocardiographic assessment may be required to optimise this setting. Indeed it may be that there are no "non-responders" as such, and providing that a suitable site for the LV lead is found optimal programming could ensure the vast majority of patients respond.

On more recent biventricular pacing devices, the output and timing of right and left ventricular stimulation are independently programmable and there is increasing interest in optimisation of the inter-ventricular delay. Sogaard and 
colleagues $^{29}$ looked at this issue in a group of 21 patients with LBBB and congestive heart failure. They concluded that while simultaneous biventricular pacing produced a predictable improvement in haemodynamic parameters, further refinement of ventricular "offset" achieved even greater benefits in systolic and diastolic performance as measured by TDI. Notably several patients in this study benefited from preactivation of the right ventricle before the left ventricle and there was considerable inter-individual variability, suggesting that individual "fine tuning" of the $\mathrm{V}-\mathrm{V}$ interval is required to gain maximal benefit. Several studies have assessed various invasive and non-invasive methods of optimising this aspect of CRT programming, including beat-beat analysis of cardiac output and haemodilutional techniques, but there are few data correlating these with long term clinical effects. Although large interventricular delays (up to $100 \mathrm{~ms}$ ) can be programmed, the optimal settings in this study were in a relatively narrow range (12-20 ms).

\section{CONCLUSION}

Clinical trials of CRT have demonstrated considerable improvements in quality of life and exercise capacity, but a significant number of "non-responders" have diluted the overall benefits. The use of ECG criteria alone will result in selection of some patients who are unlikely to benefit and also exclusion of potential responders. Assessment of regional LV mechanical activation and viability with echocardiography should be considered before implantation of a biventricular pacing device and further studies are required to refine the selection process. Once a decision has been made to proceed with CRT then appropriate placement of the LV lead and optimal programming of the device will maximise the therapeutic effect. A combination of these measures will enable CRT to deliver even more impressive results in patients with heart failure.

\section{Authors' affiliations}

D J Fox, A P Fitzpatrick, Manchester Heart Centre, Manchester Royal Infirmary, Manchester, UK

N C Davidson, North West Regional Cardiac Centre, Wythenshawe Hospital, Manchester, UK

\section{REFERENCES}

1 Abraham WT, Hayes DL. Cardiac resynchronisation therapy for heart failure. Circulation 2003; 108:2596-603.

2 Kerwin WF, Botvinick EH, O'Connell JW, et al. Ventricular contraction abnormalities in dilated cardiomyopathy: effect of biventricular pacing to correct interventricular dyssynchrony. J Am Coll Cardiol 2000:35:1221-7.

3 Auricchio A, Stellbrink C, Block M, et al. Effect of pacing chamber and atrioventricular delay on acute systolic function of paced patients with congestive heart failure. Circulation 1999;99:2993-3001.

4 Caseau S, Ritter P, Lazarus A, et al. Multisite pacing for end stage heart failure: early experience. Pacing Clin Electrophysiol 1996; 19:1748-57.

5 Daubert JC, Ritter $\mathrm{P}$, Le Breton $\mathrm{H}$, et al. Permanent left ventricular pacing with transvenous leads inserted into the coronary veins. Pacing Clin Electrophysiol 1998;21:239-45.
6 Saxon LA, Ellenbogen KA. Resynchronisation therapy for the treatment of heart failure. Circulation 2003;108:1044-8.

7 Bhatta L, Luck JC, Wolbrette DL, et al. Complications of biventricular pacing. Curr Opin Cardiol 2004;19(1):31-5.

8 Yu CM, Fung WH, Lin $\mathrm{H}$, et al. Predictors of left ventricular reverse remodelling after cardiac resynchronisation therapy for heart failure secondary to idiopathic dilated or ishaemic cardiomyopathy. Am J Cardiol 2003;91:684-8.

9 Reuter S, Garrigue S, Barold SS, et al. Comparision of characteristics in responders versus non responders with biventricular pacing for drug resistant congestive heart failure. Am J Cardiol 2002;89:346-50.

10 Abraham W. Cardiac resynchronisation therapy: a review of clinical trials and criteria for identifying the appropriate patient. Reviews in Cardiovascular Medicine 2003:4(2):S30-7.

11 Bristow MR, Saxon LA, Boehmer J, et al. Cardiac-resynchronisation therapy with or without an implantable defibrillator in advanced chronic heart failure. N Engl J Med 2004;350:2140-50.

12 Auricchio A, Stellbrink C, Sack S, et al. The pacing therapies for congestive heart failure (PATH CHF) study: rationale, design and endpoints of a prospective randomised multicentre study. Am J Cardiol 1999;83:130D-5D.

13 Abraham WT, Fisher WG, Smith A, et al. Cardiac resynchronisation in chronic heart failure. N Engl J Med 2002:346:1845-53.

14 Caseau S, Leclercq C, Lavergne T, et al. Effects of multisite biventricular pacing in patients with heart failure and intraventricular conduction delay. N Engl J Med 2001;344:873-80.

15 Sciagra R, Giaccardi M, Porciani MC, et al. Myocardial perfusion imaging using gated SPECT in heart failure patients undergoing cardiac resynchronisation therapy. J Nucl Med 2004;45:164-8.

16 Thackray S, Coletta A, Jones P, et al. Clinical trials update: highlights of the scientific sessions of heart failure 2001, a meeting of the working group on heart failure of the European Society of Cardiology. CONTAK-CD, CHRISTMAS, OPTIME-CHF. Eur J Heart Fail 2001;3:491-4.

17 Garrigue S, Reuter S, Labeque JN, et al. Usefulness of biventricular pacing in patients with congestive heart failure and right bundle branch block. Am J Cardiol 2001:88:1436-41.

18 Pitzalis MV, lacoviello M, Romito R, et al. Cardiac resynchronisation therapy tailored by echocardiographic evaluation of ventricular asynchrony. J Am Coll Cardiol 2002;40:1615-22.

19 Reuter S, Garrigue S, Barold SS, et al. Comparision of characteristics in responders versus non responders with biventricular pacing for drug resistant congestive heart failure. Am J Cardiol 2002;89:346-50.

20 Auricchio A, Yu CM. Beyond the measurement of QRS complex toward mechanical dyssynchrony: cardiac resynchronisation therapy in heart failure patients with a normal QRS duration. Heart 2004;90:479-81.

21 Lambiase PD, Rinaldi A, Hauck, et al. Non-contact left ventricular endocardial mapping in cardiac resynchronisation therapy. Heart 2004;90:44-51.

22 Pitzalis MV, lacoviello M, Romito R, et al. Cardiac resynchronisation therapy tailored by echocardiographic evaluation of ventricular asynchrony. J Am Coll Cardiol 2002;40:1615-22.

23 Yu CM, Lin H, Zhang Q, et al. High prevalence of left ventricular systolic and diastolic asynchrony in patients with congestive heart failure and normal QRS duration. Heart 2003;89:54-60.

24 Faris $\mathrm{O}$, Leclercq $\mathrm{C}$, Kato $\mathrm{R}$, et al. Assessment of electrical and mechanical function in the paced LBBB failing heart using tagged MRI and epicardial mapping. J Electrocardiol 2002;35(suppl):205.

25 de Lemos JA, McGuire DK, Drazner MH. B-Type natriuretic peptide in cardiovascular disease. Lancet 2003;362:316-22.

26 Butter C, Auricchio A, Stellbrink C, et al. Effect of resynchronisation therapy site on the systolic function of heart failure patients-on behalf of the pacing therapy for chronic heart failure II (PATH-CHF-II) study group. Circulation 2001; 104:3026-9

27 Auricchio A, Stellbrink C, Sack S, et al. Long term clinical effect of haemodynamically optimised cardiac resynchronisation therapy in patients with heart failure and ventricular conduction delay. J Am Coll Cardiol 2002;39:2026-33.

28 Sassara M, Achilli A, Ficili S, et al. Efficacy of cardiac resynchronisation therapy in narrow QRS patients [abstract]. Europace 2002;3:A29.

29 Sogaard P, Egeblad H, Pedersen AK, et al. Sequential versus simultaneous biventricular resynchronisation for severe heart failure: evaluation by tissue Doppler imaging. Circulation 2002;106:2078-84. 